\title{
Abscheideverhalten der Hochdruck-Wasserabscheider des Erdgasspeichers Berlin
}

\author{
Matthias Stark, Armin Schneider, Lothar Martini, Bernd Stark, Stephan Swientek, Rolf Nitzsche
}

\section{Einleitung}

Von der GASAG Berliner Gaswerke Aktiengesellschaft wird zur Deckung von Bedarfsspitzen in der Gasversorgung der Stadt Berlin ein Erdgas-Untertagespeicher betrieben. Bei diesem Speicher handelt es sich um einen Porenspeicher. Das Erdgas wird in einem porösen Sandsteinpaket in rund 900 Meter Tiefe unter hohem Druck gespeichert und bei Bedarf in das Gasleitungssystem der GASAG eingespeist.

Die Verbindung zum Speicher stellen mehrere Tiefbohrungen her. Die Dichtheit des Speichers wird nach oben durch eine mehrere hundert Meter mächtige Schicht undurchdringlicher Gesteine über dem Sandstein-Paket gewährleistet. Zu den Seiten hin wird das Gas durch Lagerstättenwasser, das sich vor der Einspeisung des Gases im Speichersandstein befand, begrenzt. Nach unten dichten wiederum undurchlässige Gesteine den Speicher ab. In Abbildung 1 ist der Speicher dargestellt.

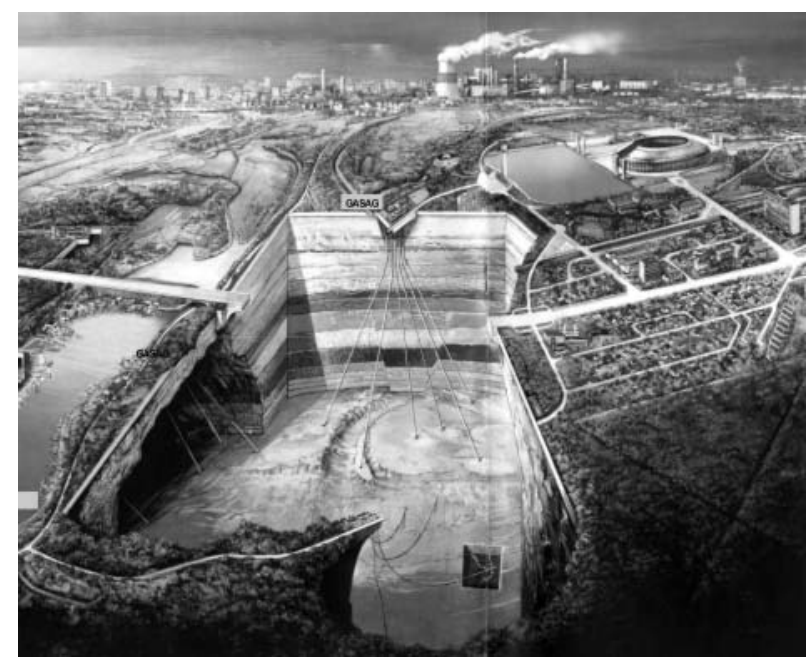

Abb. 1: Erdgas-Untertagespeicher mit Tiefbohrungen für Fördersonden

Bei der Ausspeisung des Gases aus dem Speicher wird auch Wasser mitgefördert, das sich neben dem Gas in den Poren des Sandsteins befindet, weil es nicht vollständig von Gas verdrängt wurde. Dieses Wasser muss aus dem Gas abgeschieden werden, bevor das Gas in das Berliner Gasnetz eingeleitet wird. Hierzu verfuigt der Erdgasspeicher über verfahrenstechnische Anlagen mit Hochruckabscheidern und Gastrocknungen.

\section{Ausgangssituation}

Alle Speicherbohrungen des Erdgasspeichers Berlin haben in der Gasleitung hinter dem Kopf der Bohrung einen Hochdruck-Wasserabscheider (siehe Abbildung 2).

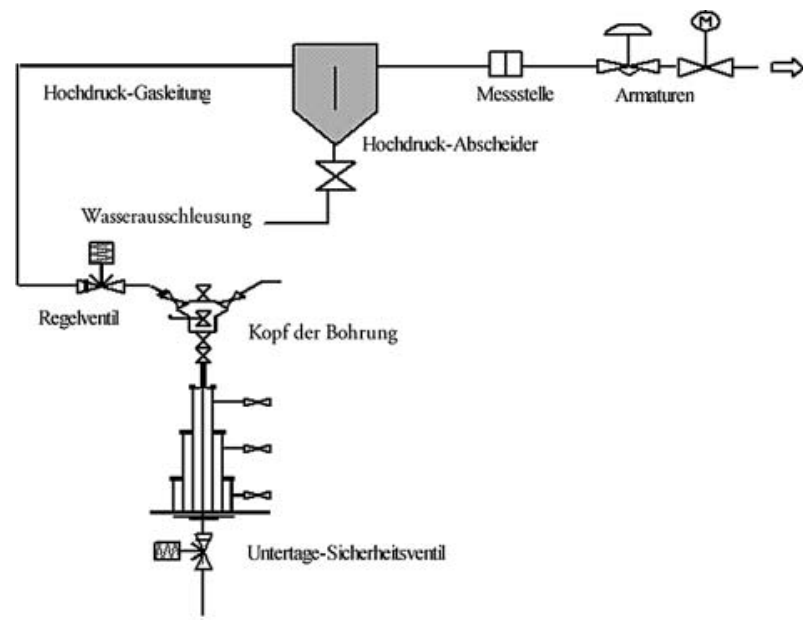

Abb. 2: Hochdruckabscheider mit Messstelle

Die Abscheideleistung der Hochdruck-Abscheider (HDAbscheider) hängt von der Durchströmgeschwindigkeit und damit von der Durchsatzrate ab. Betriebserfahrungen mit den Abscheidern deuten darauf hin, dass insbesondere bei hohen Durchsatzraten keine vollständige Abscheidung der Wasserpartikel mehr erfolgt. Es sollte daher ermittelt werden, bei welchen Durchsatzraten keine vollständige Abscheidung mehr erfolgt. Darüber hinaus war von besonderem Interesse, wie groß die nicht abgeschiedenen Partikel sind.

\section{Vorgehensweise}

Das Abscheideverhalten und die Größenverteilung der nicht abgeschiedenen Partikel wurden bei unterschiedlichen Durchsatzraten ermittelt. Um eine stabile und messbare Wasserbeladung des Gasstromes vor den Abscheidern zu gewährleisten, wurde bei lagerstättenwasserfreier Gasförderung aus dem Speicher am Kopf einer Speicherbohrung definiert Wasser zugedüst. Eine einfache Transmissionsmessung mittels einer Laser-DiodenAnordnung lieferte in Abhängigkeit von der Gasdurchsatzrate Informationen zum Vorhandensein von Wassertröpfchen im Gasstrom hinter dem HD-Abscheider. Mit Hilfe einer Partikelgrößenmessung auf der Basis des Laserbeugungsverfahrens durch das Partikelgrößenmessgerät „Spraytec“ der Firma Malvern wurde die Größenverteilung der Tröpfchen ermittelt $[1,2,3,4]$. 


\section{Messungen und Ergebnisse}

\subsection{Partikelgrößen}

\subsubsection{Messverfahren und Messaufbau}

Das Messprinzip ist in Abbildung 3 dargestellt. Es beruht auf der Beugung eines Laserstrahls durch Tröpfchen. Diese Lichtbeugung fuihrt zu einer Ablenkung von Teilen des Laserlichtes, wobei der Grad der Ablenkung von der Größe der Tröpfchen abhängt. Die Lichtintensität in der Strahlachse nimmt dadurch ab (Abfall der Transmission). Eine Transmissionsminderung zeigt an, dass sich Tröpfchen im Strahlengang des Lasers befinden. Aus einer Messung der radialen Verteilung der abgelenkten Strahlung kann die Tröpfchengrößenverteilung berechnet werden.

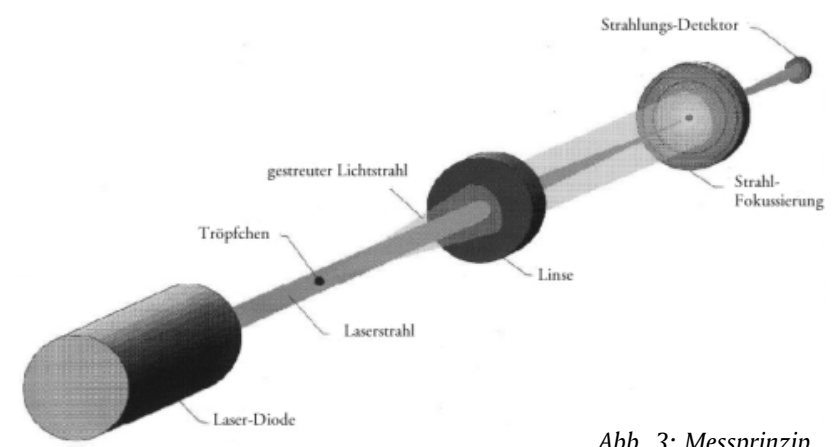

Abb. 3: Messprinzip

Um eine Durchleuchtung des Gasstromes zu ermöglichen, wurde ein Rohrstiick hinter dem HD-Abscheider ausgebaut und durch einen speziell angefertigten Fensterflansch sowie ein verkürztes Rohrstück ersetzt. In den Fensterflansch wurden gegenuiberliegende druckfeste Schaugläser eingebaut. Das Lasermessgerät für die Partikelgrößenbestimmung bzw. die Laser-Dioden-Anordnung für die Transmissionsmessung wurden am Fensterflansch montiert und justiert. Der Messaufbau ist für folgende Messparameter ausgelegt:

Gasdruck:

Strömungsgeschwindigkeit:

80 bis 120 bar Tröpfchengröße:

5 bis $25 \mathrm{~m} / \mathrm{s}$

10 bis $200 \mu \mathrm{m}$

\subsubsection{Ergebnisse der ersten Messung}

Die erste Messung mit dem „Spraytec“-Partikelgrößenmessgerät erfolgte Anfang April 2000. Zu diesem Zeitpunkt betrug der Druck am HD-Abscheider der Speicherbohrung ca. 100 bar. Die Durchsatzrate durch den Abscheider wurde auf $25.000 \mathrm{~m}^{3}(\mathrm{Vn}) / \mathrm{h}$ eingestellt. Dies entspricht etwa $250 \mathrm{~m}^{3}(\mathrm{~B}) / \mathrm{h}$. * Vor dem HD-Abscheider (am Sondenkopf) wurde Wasser mit einer zeitlich konstanten Rate von ca. 300 l/h zugedüst.

Bei der ersten Messung mit dem Partikelgrößenmessgerät wurde unmittelbar nach dem Anfördern der Speicherbohrung das Auftreten von Tröpfchen festgestellt. Die Fenster, die zum Durchleuchten des Gasstromes in der Gasleitung angebracht waren, verschmutzten sehr schnell. Dadurch wurde das Messsignal bereits nach kurzer Zeit so klein, dass die Messung abgebrochen werden musste. In der relativ kurzen Messzeit konnten jedoch genügend Daten aufgezeichnet werden, um eine erste grobe Auswertung vorzunehmen. Diese Auswertung ergab, dass der Gasstrom Tröpfchen mit einem Durchmesser von etwa 8 bis $80 \mu \mathrm{m}$ Durchmesser enthielt. Das Maximum der Verteilung lag bei ungefähr 40 $\mu \mathrm{m}$. Eine Darstellung der gemessenen Tröpfchenverteilung findet sich in Abbildung 4.

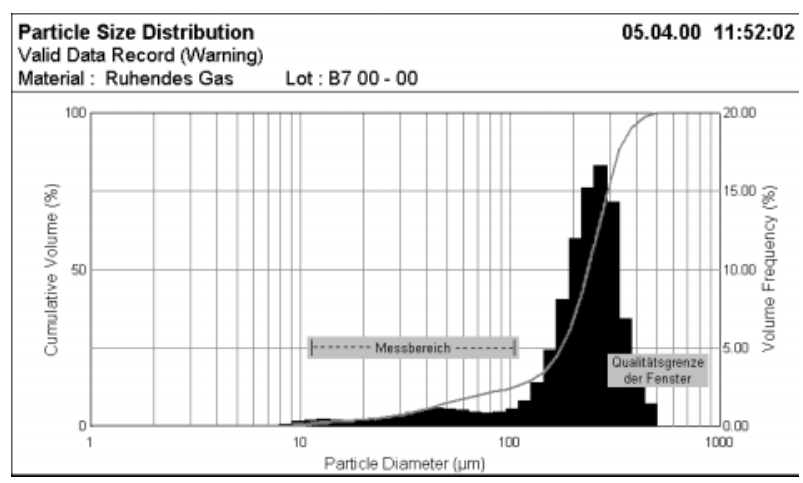

Abb. 4: Tröpfchengrößenspektrum der ersten Messung mit „Spraytec“

Der Niederschlag auf den Fenstern verdunstete nicht. Es musste daher davon ausgegangen werden, dass das Messergebnis stark von den Verschmutzungen auf den Fenstern beeinflusst wurde.

Der hohe Anteil von Teilchen mit Durchmessern größer $100 \mu \mathrm{m}$ wird durch Einschliusse im Fenster verursacht, nicht durch Tröpfchen. Da mit Tröpfchen größer als $100 \mu \mathrm{m}$ nicht gerechnet werden musste, war diese Fensterqualität ausreichend [5].

Nach Ausbau der Fenster wurde festgestellt, dass es sich bei den Verschmutzungen um einen zum Teil öligen Niederschlag handelte. Es waren also zumindest nicht ausschließlich Wassertröpfchen, sondern auch Öl- oder Kondensattröpfchen im Gas, die vermutlich aus dem Abscheider mitgerissen worden sind.

Um eine starke Niederschlagsbildung auf den Fenstern bei weiteren Messungen zu vermeiden, wurde der HDAbscheider zunächst gesäubert, so dass vorhandene Öl-, Fett- oder Kondensatrüickstände nicht vom Gasstrom mitgerissen werden konnten. Darüber hinaus wurden alle weiteren Messungen in einem Temperatur- und Druckbereich durchgefuihrt, in dem eine Tröpfchenbildung durch Kondensation höherwertiger Kohlenwasserstoffe aus dem Gasstrom weitestgehend vermieden wurde.

Vor einer erneuten Messung der Partikelgrößenverteilung wurde eine Zwischenmessung durchgefuihrt, die zunächst nur zeigen sollte, ob bzw. wann Wassertröpfchen nicht mehr vollständig abgeschieden werden. Diese Messung lieferte keine Information über vorhandene Tröpfchendurchmesser, war aber weniger anfällig gegenüber einer Verschmutzung der Fenster.

Die Zwischenmessung erfolgte Anfang April 2000. Aufgrund der geringeren Schmutzanfälligkeit konnte über einen relativ langen Zeitraum gemessen werden. 


\subsection{Transmissionsmessung}

\subsubsection{Messaufbau und Messverfahren}

Zur Realisierung einer optischen Transmissionsmessung durch die speziell gefertigte Hochdruck-Messkuivette wurden ein Halbleiterlaser als Strahlungsquelle (unmoduliert; $1 \mathrm{~mW}$ Strahlungsleistung; $670 \mathrm{~nm}$ Wellenlänge; Strahlfläche $2 \times 1 \mathrm{~mm}^{2}$ ) und eine Photodiode als Strahlungsempfänger mit integrierter Verstärkerschaltung (Typ Newport 818BB) gegenüberliegend außerhalb der Messküvette aufgebaut. Laser und Photodiode waren über eine $x$-y-z-Positioniervorrichtung und eine Winkelverstelleinheit gegeneinander justierbar. Diese Anordnung ermöglichte die Einstellung eines Strahlweges der Laserstrahlung durch die Fenster der Messküvette, der frei war von optischen Defekten in den Küvetten-Fenstern. Durch die Feinjustagemöglichkeit von Strahlungsquelle und Strahlungsempfänger im Zusammenhang mit den Schichten zur Entspiegelung der Küvettenfenster konnte weiterhin die Entstehung von Interferenzstrukturen durch Reflexion an den Fensteroberflächen vermieden werden. Diese Interferenzstrukturen würde der Partikelanalysator als Beugung an Partikeln deuten. Auf diese Weise war eine störungsfreie Transmissionsmessung durch die Messküvette gewährleistet. Signaländerungen des Strahlungsempfängers waren so nur Strahlungsleistungsänderungen durch Vorgänge innerhalb der Küvette zuzuordnen. Durch eine spezielle Blendenanordnung im Messstrahlengang empfing der Strahlungsempfänger nur die direkte, extrem in Vorwärtsrichtung gestreute Laserstrahlung. In Verbindung mit der Messwellenlänge war garantiert, dass die Extinktion der Strahlung auf reine Streuung an Wassertröpfchen im Strahlengang und nicht auf zusätzliche Absorption zurückzufuihren ist. Die Aufzeichnung der Messdaten erfolgte mit einem 16 bit-Datenlogger hoher Samplingrate.

Bei Vorversuchen im Labor wurde in einem drucklosen Messaufbau mit Laser und Photodiode sowie im Strahlengang justierten Küvettenfenstern mit Hilfe unterschiedlicher künstlicher Wassernebel der Zusammenhang zwischen Nebeldichte, Tröpfchengröße und Signalgröße des Strahlungsempfängers nachgewiesen. Es wurde weiterhin nachgewiesen, dass die Drift der Ausgangsleistung des verwendeten Diodenlasers bei annähernd konstanten Umgebungsbedingungen zu vernachlässigen ist.

\subsubsection{Ergebnisse der Zwischenmessung}

In der Zwischenmessung wurde nur die Transmission des Laserlichtes gemessen. Ein Abfall des Messsignals zeigte also an, dass sich Tröpfchen im Gasstrom befanden. Zum Zeitpunkt der Messung betrug der Druck am HDAbscheider der Speicherbohrung etwa 120 bar. Auf ein Heizen des Fensterflansches wurde verzichtet.

Nach einer Nullmessung (trockene Gasförderung, $\left.20.000 \mathrm{~m}^{3}(\mathrm{Vn}) / \mathrm{h}=170 \mathrm{~m}^{3}(\mathrm{~B}) / \mathrm{h}\right)$ wurden zunächst verschiedene Durchsatzraten von 20.000 bis $55.000 \mathrm{~m}^{3}(\mathrm{Vn}) / \mathrm{h}$ (170 bis $\left.515 \mathrm{~m}^{3}(\mathrm{~B}) / \mathrm{h}\right)$ eingestellt. Hierbei erfolgte am Sondenkopf vor dem HD-Abscheider eine zeitlich konstante Wasserzuduisung von 300 bzw. 500 l/h (1 bis 6 l
Wasser auf $\left.1 \mathrm{~m}^{3}(\mathrm{~B}) \mathrm{Gas}\right)$. Aus der Lagerstätte wurde kein Wasser mitgefördert.

Die Messung der Transmission erfolgte kontinuierlich, wobei ab einer Rate von $260 \mathrm{~m}^{3}(\mathrm{~B}) / \mathrm{h}$ und einer Wasserbeladung von ungefähr $2 \mathrm{l} / \mathrm{m}^{3}(\mathrm{~B})$ erste deutliche Anzeichen von Tröpfchenanfall hinter dem HD-Abscheider messbar waren. Bei Steigerung der Gasraten nahm auch die Transmission deutlich ab. Hierbei ist jedoch zu berücksichtigen, dass die Messung durch einen zunehmenden Niederschlag auf den Fenstern beeinflusst wurde.

Bei einer Gasrate von $55.000 \mathrm{~m}^{3}(\mathrm{Vn}) / \mathrm{h}=514 \mathrm{~m}^{3}(\mathrm{~B}) / \mathrm{h}$ wurde die Wasserzudüsung gestoppt. Mit geringer zeitlicher Verzögerung (Wegstrecke Sondenkopf - HD-Abscheider) nahm auch die Transmission wieder zu, wobei die ursprüngliche, während der Nullmessung gemessene Signalhöhe nicht mehr erreicht wurde. Dies war auf den Niederschlag, der sich auf den Fenstern gebildet hatte, zurückzufuihren.

Im weiteren Verlauf der Messung wurden wiederum konstante Gasraten von 55.000 bis $10.000 \mathrm{~m}^{3}(\mathrm{Vn}) / \mathrm{h}(515$ bis $\left.85 \mathrm{~m}^{3}(\mathrm{~B}) / \mathrm{h}\right)$ eingestellt. Die Wasserzudüsung erfolgte jetzt aber bei jeder eingestellten Gasrate nur jeweils eine Minute lang (peakartige Zuduisung). Bei allen Raten wurde ein entsprechender Transmissions-Abfall gemessen. Die zeitliche Verzögerung vom Einsetzen der Zudüsung bis zum Abfallen der Transmission war hierbei abhängig von der Durchsatzrate (Strömungsgeschwindigkeit). Aufgrund der peakartigen Wasserzudüsung war die weitere Niederschlagsbildung auf den Fenstern nur noch gering.

Der Messverlauf mit Messsignal der Photodiode [V] (Transmissionsintensität), Durchsatzraten [Betriebskubikmeter/Std.] und Wasserzudüisung [Liter/Betriebskubikmeter-Gas] ist in Abbildung 5 dargestellt.

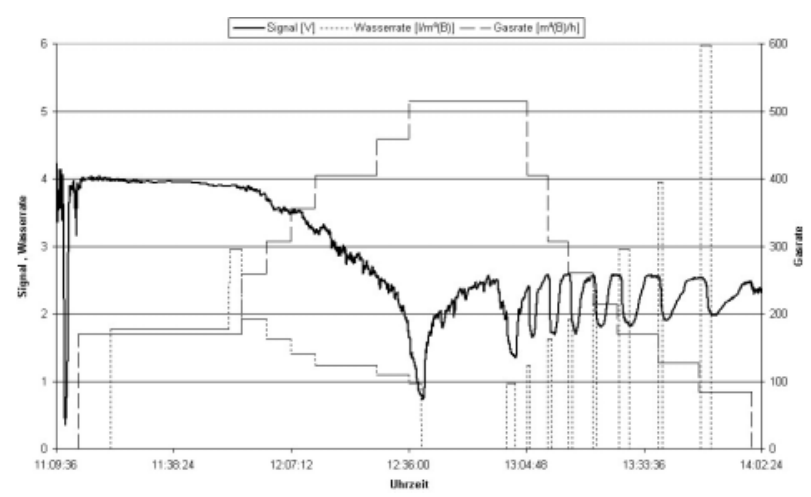

Abb. 5: Darstellung des Verlaufes der Transmissionsmessung

Wie der Messverlauf zeigt, tritt zum Ende der Messreihe bei Durchsatzraten von $10.000 \mathrm{~m}^{3}(\mathrm{Vn}) / \mathrm{h}\left(85 \mathrm{~m}^{3}(\mathrm{~B}) / \mathrm{h}\right)$ und peakartiger Zuduisung von $500 \mathrm{l} / \mathrm{h}\left(6 \mathrm{l} / \mathrm{m}^{3}(\mathrm{~B})\right)$ im Gegensatz zum Messbeginn noch ein Transmissionsabfall auf. Es sind also noch Tröpfchen im Gasstrom vorhanden. Dies ist höchstwahrscheinlich darauf zurückzuführen, dass die Wasserbeladung des Gasstromes extrem hoch ist $\left(6 \mathrm{l} / \mathrm{m}^{3}(\mathrm{~B})\right)$, wobei das Wasser durch die Zuduisung sehr fein vernebelt sein dürfte. Mit solchen extremen Verhältnissen ist im Betrieb nicht zu rechnen. 
Während der kontinuierlichen Zudüsung von Wasser sank das Messsignal kontinuierlich ab und stieg nach Beendigung der Wasserzuduisung nur auf rund 60 Prozent des Ausgangswertes an. Der Abfall des Messsignals wurde durch einen leichten Niederschlag auf den Fenstern verursacht. Bei der nachfolgenden peakartigen Zudüsung des Wassers verstärkte sich der Niederschlag nicht mehr. Nach Abbruch der jeweils kurzen Zudüsungen (Peaks), wurde das Ausgangssignal der trockenen Gasförderung wieder erreicht.

Auf Grundlage der so erzielten Messergebnisse konnten zunächst folgende qualitative Aussagen gemacht werden:

- Bei Gasdurchsatzraten ab ca. $250 \mathrm{~m}^{3}(\mathrm{~B}) / \mathrm{h}$ und moderater Wasserbeladung des Gasstromes (ca. $2 \mathrm{l} / \mathrm{m}^{3}(\mathrm{~B})$ ) wird das Wasser nicht mehr vollständig abgeschieden.

- Be höherer Wasserbeladung (4 l/ $\left.\mathrm{m}^{3}(\mathrm{~B})\right)$ wird auch bei geringeren Gasraten das Wasser nicht mehr vollständig abgeschieden.

Da sich bei der peakartigen Wasserzuduisung nach den jeweiligen Peaks wieder reproduzierbare Messverhältnisse einstellten, wurde versucht, aus den Messergebnissen auch quantitative Aussagen abzuleiten. Die Vorgehensweise hierzu ist in Folgendem beschrieben.

Aus den Signalhöhen an der Photodiode wurden Koeffizienten errechnet, die einen quantitativen Ausdruck des Abscheideverhaltens liefern:

- Die Signalhöhe an der Photodiode während der peakartigen Wasserzuduisung vor dem Abscheider wurde ins Verhältnis zur Signalhöhe während trockener Gasförderung gesetzt, um ein Maß für Tröpfchenbeladung des Gasstromes hinter dem Abscheider bei Wasserzuduisung zu erhalten.

- Die Wasserzuduisung erfolgte mit konstanter Rate, während die Gasrate variiert wurde. Daraus ergaben sich in Abhängigkeit von der eingestellten Gasrate unterschiedliche Wasserbeladungen des Gasstromes vor dem Abscheider. Um dies zu berücksichtigen, wurde das o. a. Signalverhältnis auf die kleinste Wasserbeladung normiert.

Bei der quantitativen Auswertung wurden Koeffizienten ermittelt, die das Abscheideverhalten der HD-Abscheider in etwa beschreiben sollen. Sie sind in Tabelle 1 aufgelistet.

\begin{tabular}{|c|c|c|}
\hline Gasrate $\left[\operatorname{urt}^{7}(\mathrm{Vni}) / \mathrm{h}\right]$ & Gasrate $\left[\operatorname{rr}^{\top}\left(\mathrm{B}^{\prime}\right) / \mathrm{h}\right]$ & Koeffizient Abscheidume \\
\hline 10.000 & 85 & 0,9 \\
\hline 20.000 & 170 & 0,45 \\
\hline 30.000 & 260 & 0,3 \\
\hline 45.000 & 405 & 0,15 \\
\hline 5.000 & 515 & 0,1 \\
\hline
\end{tabular}

Tab. 1: Abscheidekoeffizienten

Diese Koeffizienten sind keine Abscheidegrade, da sie nicht auf der Messung von Wassermengen vor bzw. hinter dem Abscheider basieren, sondern nur aus Signalveränderungen aufgrund der Tröpfchenbeladung des Gasstromes resultieren. Der Zusammenhang zwischen Signalveränderung und Tröpfchenbeladung ist nicht bekannt. Die Koeffizienten liefern aber in jedem Fall Anhaltspunkte für das Abscheideverhalten: Je kleiner der Koeffizient, desto geringer der Abscheidegrad. Die Abscheidekoeffizienten sind in Abbildung 6 über dem Gasdurchsatz durch den Hochdruckabscheider dargestellt.

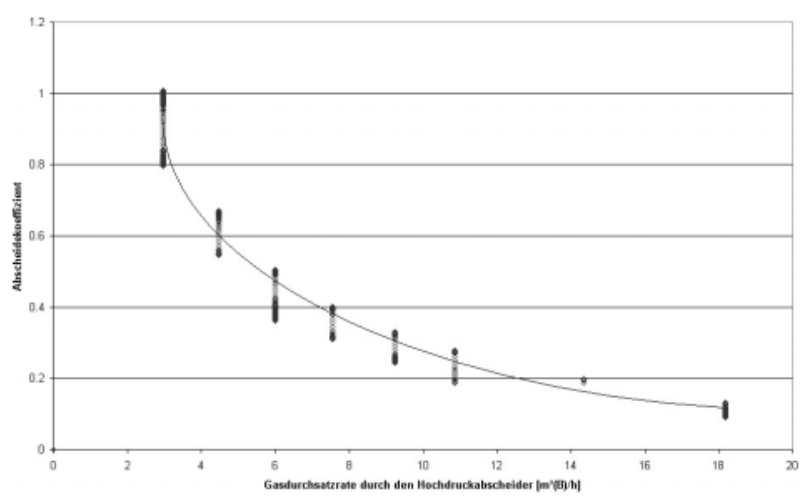

Abb. 6: Darstellung des Abscheideverhaltens des Hochdruckabscheiders in Abhängigkeit vom Durchsatz

\subsection{Partikelgrößen - Ergebnisse der zweiten Messung}

Aufgrund der Erfahrungen aus der Zwischenmessung wurde die zweite Messung mit dem Laser-Partikelgrößenmessgerät ebenfalls mit peakartiger Wasserzuduisung durchgefuihrt.

Die zweite Partikelgrößenmessung fand im Februar 2001 statt. Die Messanordnung entsprach der ersten Messung. Aufgrund der optimierten Messdurchfuihrung (peakartige Zuduisung von jeweils $500 \mathrm{l} / \mathrm{h}$ für 1 bis 2 Minuten) konnte wesentlich länger als beim ersten Mal gemessen werden. Insgesamt wurden bei fünf verschiedenen Durchsatzraten die Partikelgrößen im Gasstrom hinter

\begin{tabular}{|c|c|c|c|}
\hline \begin{tabular}{|l|} 
FE- \\
Zei
\end{tabular} & 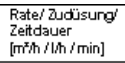 & Messergetris & Eemerkingen \\
\hline $\begin{array}{l}11: 35 \\
11: 55\end{array}$ & $\begin{array}{l}10.000 / 0 \\
10.000 / 500 / 1\end{array}$ & $\begin{array}{l}\text { Aackground unweraindert } \\
\text { Background kaum werändert }\end{array}$ & wallstindige thscheidung \\
\hline 12:05 & $30.000 / 500 / 1$ & 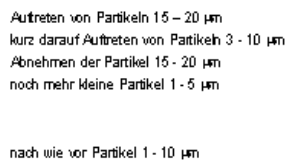 & 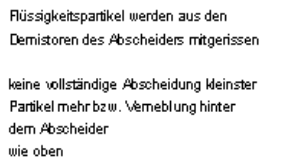 \\
\hline $\begin{array}{l}12 \% 0 \\
1230\end{array}$ & $\begin{array}{l}50.000 / 0 \\
50.000 / 0 / 1 \\
50.000 / 0 / 1\end{array}$ & 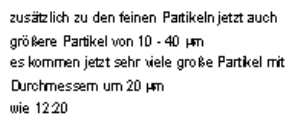 & 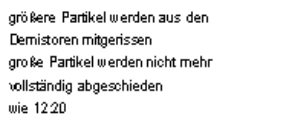 \\
\hline 13:00 & $\begin{array}{l}30.000 / 500 / 2 \\
30.000 / 0\end{array}$ & 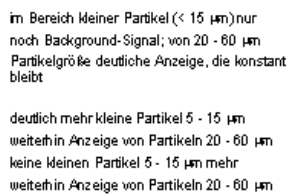 & 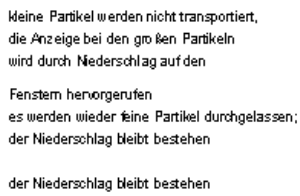 \\
\hline $13: 08$ & $40.000 / 500 / 2$ & 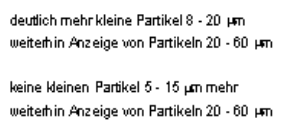 & 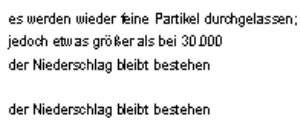 \\
\hline $\begin{array}{l}13: 10 \\
13: 15\end{array}$ & $\begin{array}{l}20.000 / 0 \\
20.000 / 500 / 2\end{array}$ & 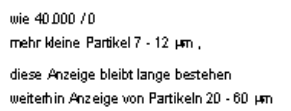 & 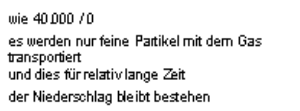 \\
\hline $13: 25$ & Sonde alu & 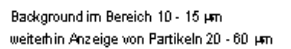 & $\begin{array}{l}\text { heine Partikel mehri in Gizs } \\
\text { der Niederschlag tleibt bettehen }\end{array}$ \\
\hline
\end{tabular}

Tab. 2: Messverlauf 
dem Abscheider ermittelt. Der Messverlauf mit den Ergebnissen ist in Tabelle 2 detailliert aufgezeichnet.

In den folgenden Abbildungen 7 und 8 sind Beispiele für die gemessenen Partikelgrößenverteilungen dargestellt. Die Zeiten entsprechen dabei ungefähr den Zeiten in der obigen Tabelle.

In Abbildung 7 ist ein zweites Maximum der Partikelgrößenverteilung bei rund $80 \mu \mathrm{m}$ zu erkennen, das nicht von Tröpfchen herrührt, sondern von einer optischen Beeinträchtigung (Linsenwirkung) der stahlummantelten Schaugläser hervorgerufen wurde. Dieser Effekt war während der Messung nicht zu eliminieren. Er beeinflusste die Auswertbarkeit jedoch nicht, da die auftretenden Tröpfchen kleiner waren und sich deutlich von diesem Maximum absetzten.

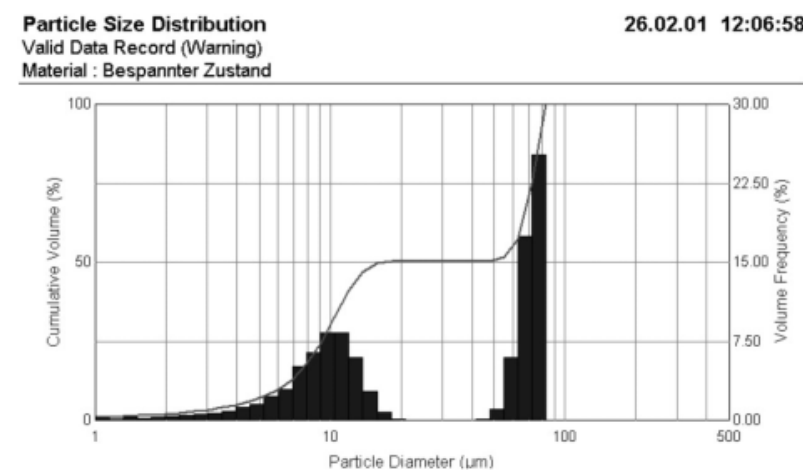

Abb. 7: Partikelgrößenverteilung bei $30.000 \mathrm{~m}^{3}(\mathrm{Vn})$ Gasrate und $500 \mathrm{l} / \mathrm{h}$ Zudüsung

Abbildung 8 zeigt die Partikelgrößenverteilung bei einer Gasrate von $50.000 \mathrm{~m}^{3}(\mathrm{Vn}) / \mathrm{h}$. Gut zu erkennen ist die Verschiebung des Größenspektrum hin zu größeren Tröpfchendurchmessern.

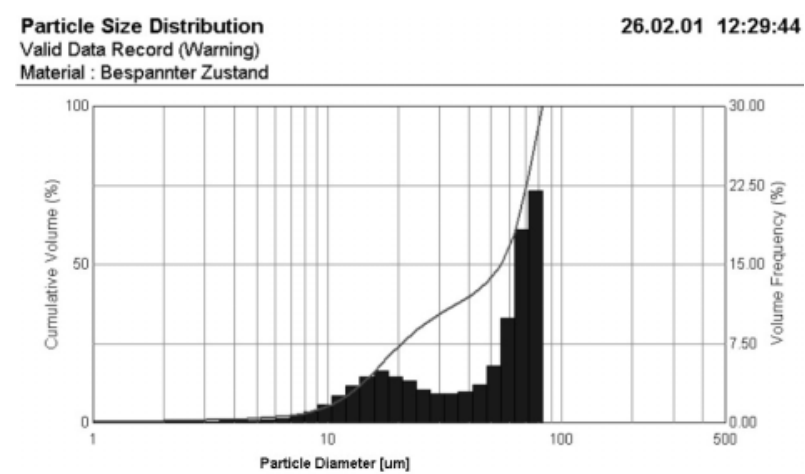

Abbildung 8: Partikelgrößenverteilung bei $50.000 \mathrm{~m}^{3}$ (Vn) Gasrate und $500 \mathrm{l} / \mathrm{h}$ Zudüsung

Nachfolgend sind die Partikel-Messergebnisse tabellarisch zusammengefasst.

\begin{tabular}{|c|c|c|}
\hline Gasrate $\left[\mathrm{mr}^{7}(\mathrm{~W}) / \mathrm{h}\right]$ & Fartikeläalie [um] & Maxirnum $[\lceil\mathrm{mm}]$ \\
\hline 10.000 & & \\
\hline 20.000 & bis 12 & 10 \\
\hline 30.000 & bis 15 & 10 \\
\hline 40.000 & $b i=20$ & 15 \\
\hline 50.000 & bi $=40$ & 20 \\
\hline
\end{tabular}

Tab. 3: Partikel-Messergebnisse

Die Messergebnisse der zweiten Partikelgrößenmessung stehen damit im Einklang mit der Zwischenmessung. Es wird deutlich sichtbar, dass mit höheren Gasraten immer größere Teilchen nicht mehr vollständig abgeschieden werden bzw. mit dem Gasstrom aus dem Abscheider mitgerissen werden. Gegenüber der ersten Messung mit dem Partikelgrößenmessgerät sind die bei Förderraten von 20.000 bis $30.000 \mathrm{~m}^{3}(\mathrm{Vn}) / \mathrm{h}\left(85 \mathrm{bis} 260 \mathrm{~m}^{3}(\mathrm{~B}) / \mathrm{h}\right)$ gemessenen Tröpfchengrößen kleiner. Hierbei ist jedoch zu berücksichtigen, dass bei der ersten Messung vermutlich sehr viele Verunreinigungen aus dem Abscheider mitgerissen wurden, die vermutlich zu einem höheren Anteil größerer Partikel im Gas gefuihrt haben.

\subsection{Weitere Erfahrungen}

Da die Messungen im laufenden Betrieb des Erdgasspeichers durchgefuihrt wurden, waren die Anforderungen an die Messplanung und Vorbereitung hoch. Insbesondere der Messaufbau stellte eine große Herausforderung dar, da in die Gasleitung hinter dem Abscheider druckfeste Schaugläser eingebaut werden mussten, um den Gasstrom mit dem Laser zu durchstrahlen. Da mit einem relativ weiten Laserstrahl gearbeitet wurde, mussten die Gläser große Durchmesser haben, was bei einem Prüfdruck von 180 bar zu entsprechend großen, notwendigen Glasdicken fuihrte. Darüber hinaus mussten die Gläser über ausreichend gute optische Eigenschaften verfügen und entspiegelt sein. Die Entspiegelung war notwendig, weil Interferenzstrukturen durch Reflexion an den Fensteroberflächen vermieden werden mussten. Diese Interferenzstrukturen wären vom Partikelanalysator als Beugung an Partikeln gedeutet worden.

Neben der Verschmutzung der Gläser, die insbesondere bei den Messungen mit dem Laser-Partikelgrößenmessgerät die nutzbaren Messzeiten deutlich einschränkte und zu äußerst zügigem Messen zwang, erwies sich auch der gasdichte Einbau der Fenster in die Gasleitung als schwierig. Zunächst wurden normale Quarzglas-Schaugläser verwendet, die mit entsprechenden Dichtungen in einen speziell angefertigten Flansch der Gasleitung eingebaut wurden. Aufgrund der hohen Flächenpressungen im Dichtbereich wurden die Gläser jedoch immer wieder beschädigt und konnten nicht wieder verwendet werden. Dieses Problem konnte erst gelöst werden, nachdem die normalen Schaugläser durch spezielle Gläser mit Stahlrand ersetzt wurden, die nun den hohen Flächenpressungen im Dichtbereich standhielten.

\section{Zusammenfassung}

In einem Pilotversuch wurde in Zusammenarbeit der GASAG Berliner Gaswerke Aktiengesellschaft mit der Technischen Fachhochschule Wildau das Abscheideverhalten von Hochdruck-Wasserabscheidern des Erdgasspeichers Berlin untersucht.

Bei diesen Untersuchungen wurden die Partikelgrößenverteilungen im Gasstrom hinter dem Hochdruckabscheider der Fördersonde bei unvollständiger Abscheidung mit einem Laser-Partikel-Messgerät „Spraytec“ der Firma Malvern auf der Basis der Mie-Streuung gemessen. 
Um die Durchstrahlung des Erdgases im Rohr bei Gasdrücken von bis zu 120 bar zu ermöglichen, wurde ein spezielles Rohrzwischenstuick entwickelt, das mit gegenüberliegenden, druckfesten Spezialgläsern (Antireflexionsschichten) versehen war. Um definierte Messbedingungen zu gewährleisten, wurde vor dem HD-Abscheider am Sondenkopf Wasser in verschiedenen, zeitlich konstanten Raten (von $300 \mathrm{l} / \mathrm{h}$ bis $500 \mathrm{l} / \mathrm{h}$ ) in die trockene Gasförderung zugedüst (bei Durchsatzraten zwischen $10000 \mathrm{~m}^{3}(\mathrm{Vn}) / \mathrm{h}$ und $\left.55000 \mathrm{~m}^{3}(\mathrm{Vn}) / \mathrm{h}\right)$.

Mit einer weiteren Messanordnung, die als wesentliche Bauteile eine Laserdiode und eine Photodiode besaß, wurde bei den oben beschriebenen Messbedingungen die Strahlungstransmission durch das Erdgas (Hochdruck) gemessen.

Da alle Messungen im laufenden Betrieb des Erdgasspeichers durchgeführt werden mussten, waren die technischen Anforderungen an die Messplanung, Vorbereitung und Ausfuihrung der Untersuchungen sehr groß.

Es wurden wichtige Erkenntnisse für den technischen Betrieb von Hochdruck-Wasserabscheidern gewonnen. Von besonderer Bedeutung ist dabei eine Darstellung des Abscheidekoeffizienten über dem Gasdurchsatz durch den Hochdruckabscheider.

\section{Anmerkung}

* Bei der überschlagsmäßigen Umrechnung des Normvolumenstromes $\left(\mathrm{m}^{3}(\mathrm{Vn}) / \mathrm{h}\right)$ in den Betriebsvolumenstrom $\left(\mathrm{m}^{3}(\mathrm{~B}) / \mathrm{h}\right)$ muss der Normvolumenstrom, der in der Regel von den Gasmesseinrichtungen angezeigt wird, durch den Betriebsdruck in der Gasleitung dividiert werden. Dieser Druck ist abhängig von der Förderrate der Speicherbohrung, vom Zustand der Speicherbohrung und dem Füllstand des Erdgasspeichers.

Die Betriebsvolumenströme können sich bei gleichen Normvolumenströmen daher unterscheiden. Darüber hinaus gibt es keinen linearen Zusammenhang zwischen Normvolumenstrom und Betriebsvolumenstrom, da sich der Betriebsdruck nicht linear mit der Förderrate ändert. In die Berechnung des Normvolumenstromes geht auch noch die Gastemperatur ein. Sie wird hier aber vernachlässigt.

\section{Literatur}

1 Firmenschrift Malvern Instruments, Real Time, High Speed, High Concentration Spray Droplet Sizer, Spraytec

2 Malvern News, Ausgabe 14, März 1998, Partikelgröße in Sprays - Spraytec RTS 5000 Serie

3 Walther, Christian: Grobes in Feinem: Eine Herausforderung in der Partikelmeßtechnik, Malvern News, Ausgabe 16. Juni 1999

4 Firmenschrift Malvern Instruments, Spraytec RTS 5000 Serie, Partikel im Laserlicht

5 Abscheideleistungen der HD-Abscheider, FGT-Nr. K0048/99/ 00, Filtan Gas Technologie GmbH 1999

\section{Autoren}

Dipl.-Ing. Matthias Stark

Dipl.-Berging. Armin Schneider

GASAG Berliner Gaswerke Aktiengesellschaft

Betrieb Speicher

Glockenturmstraße 18, 14053 Berlin

Telefon +493078722654

E-Mail: mstark@gasag.de

\section{Prof. Dr. sc. nat. Bernd Stark}

Dr. habil. Lothar Martini

Dipl.-Ing. (FH) Stephan Swientek

Technische Fachhochschule Wildau

Fachbereich Ingenieurwesen/Wirtschaftsingenieurwesen

Labor für Sensortechnik/Gasanalytik

Telefon +493375 508-524

E-Mail: bstark@igw.tfh-wildau.de

\section{Dr. habil. Rolf Nitzsche}

Ingenieurbüro Dr. Nitzsche Partikelmesstechnik

Waldstraße 60, 02681 Kirschau

Telefon +49359233020 